\title{
Sarcoidosis Masquerading as Long-Standing Cholestasis
}

\author{
Smit S. Deliwala a, d, Murtaza Hussain ${ }^{\mathrm{a}}$, Anoosha Ponnapallia, Rupesh Khanal ${ }^{\mathrm{a}}$, Hemant Goyal ${ }^{\mathrm{b}}$, \\ Adil Abdalla ${ }^{\mathrm{c}}$, Mamoon M. Elbedawi ${ }^{\mathrm{c}}$
}

\begin{abstract}
Hepatic sarcoidosis is an exceedingly rare extrapulmonary manifestation of sarcoidosis, with the majority remaining stable for years without clinical clues, only displaying biochemical abnormalities. Amongst the literature, the timeline to cirrhosis has not been parsed out; hepatomegaly develops in 50\% and cirrhosis in 33\% of all hepatic sarcoidosis patients, making this an essential issue in this patient population. Interestingly, the risk for hepatocellular carcinoma remains high regardless of cirrhosis development. Corticosteroids and biologics remain the mainstay of therapy, although refractory cases may require deeper immunosuppression. Liver transplantation is seen in a handful of cases with promising results. We present an interesting case of cholestatic pattern livery injury in our outpatient setting that was eventually discovered to be hepatic sarcoidosis. Mild biochemical derangements or sole elevations in alkaline phosphatase are under-recognized, and patients often progress to cirrhosis and end-stage liver disease. This diagnostic miss has significant implications and represents an opportunity to treat liver disease with a reversible cause. Consensus guidelines recommend alkaline phosphatase screening in newly diagnosed cases of sarcoidosis.
\end{abstract}

Keywords: Sarcoid; Extrapulmonary; Transaminitis; Cholestasis; Liver

\section{Introduction}

Sarcoidosis, initially considered an aberrant form of tuberculosis, is a systemic disorder characterized by granuloma collections. Its incidence nears 8.8 per 100,000 per year and is more commonly seen in African Americans with a peak age of

Manuscript submitted January 25, 2021, accepted March 5, 2021

Published online April 21, 2021

aDepartment of Internal Medicine, Michigan State University at Hurley Medical Center, Flint, MI, USA

bDivision of Gastroenterology, Department of Internal Medicine, The Wright Center for Graduate Medical Education, Scranton, PA, USA

'Division of Gastroenterology, Department of Internal Medicine, Michigan State University at Hurley Medical Center, Flint, MI, USA

${ }^{\mathrm{d} C o r r e s p o n d i n g ~ A u t h o r: ~ S m i t ~ S . ~ D e l i w a l a, ~ D e p a r t m e n t ~ o f ~ I n t e r n a l ~ M e d i c i n e, ~}$ Michigan State University at Hurley Medical Center, One Hurley Plaza, Flint, MI 48503, USA. Email: deliwal1@msu.edu

doi: https://doi.org/10.14740/gr1360
40 - 50 years, while familial clustering is an evolving concept [1]. Sarcoidosis is primarily a lung disease with a broad array of clinical presentations, with extrapulmonary manifestations in $30 \%$ of cases. Amongst involved sites, the liver and spleen are seen infrequently, while the finding of hepatosplenomegaly is even rarer [2]. Biochemical abnormalities are seen in a fraction of patients, with many remaining asymptomatic for decades until decompensation occurs. The majority of the data on hepatic sarcoidosis come from antemortem studies, possibly overestimating symptomatology rates as many with hepatic sarcoidosis are often not diagnosed. Hepatic sarcoidosis appears to have the ability to progress to cirrhosis, although its portal hypertensionrelated complications and clinical course is different from its classical counterpart; hence, these patients require individualized care [3]. Diagnostic criteria have not been set, and the American Thoracic Society (ATS) requires granulomatous lesions in more than one organ system, ruling out other diseases and confirmation using clinical, laboratory, and radiological findings. Position statements on surveillance schedule lack and testing often occur due to clinical deterioration. The paucity of adequately powered trials in these patients makes management arbitrary in the absence of formalized guidelines [4]. Hence we decided to write a case report on this presentation.

\section{Case Report}

A 47-year-old male presented to our clinic to establish care; he had not seen a healthcare provider in over 10 years. His only complaints were fatigue, dry cough, and night sweats that had been evolving over several months, although he was told that he had briefly turned "yellow" a few years prior. He denied having a medical history, prescription or herbal formulations, or the use of tobacco, alcohol, or illicit substances. He had an 8-year stint in the US Army and denied any medical problems or radiation exposures. He was hemodynamically stable, and the physical exam was unremarkable.

\section{Investigations}

Initial workup revealed severe cholestasis and derangements in hepatobiliary indices. Hepatitis, human immunodeficiency virus (HIV), and autoimmune panels were negative. Subsequent chest and abdomen computed tomography (CT) with intravenous contrast revealed enlarged mediastinal lymph nodes, with the largest measuring $1.8 \times 1.5 \mathrm{~cm}$ in the right 


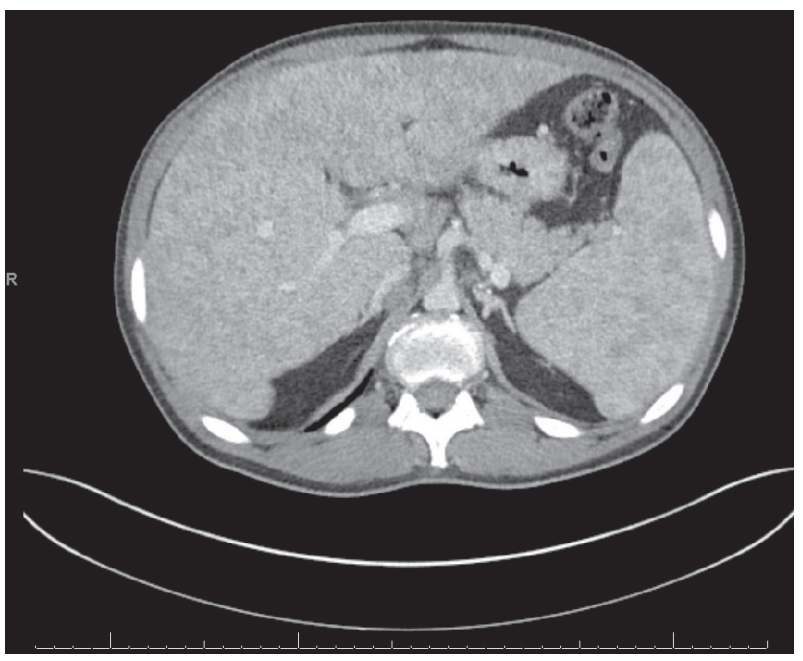

Figure 1. Computed tomography (CT) of the abdomen revealing changes consistent with cirrhosis and splenomegaly.

paratracheal region and $2.8 \times 2.0 \mathrm{~cm}$ in the subcarinal region. Heterogenous attenuation with a nodular surface suggested liver cirrhosis and co-existent splenomegaly (Fig. 1). Another feature consistent with portal hypertension seen was chronic thrombocytopenia. Bronchoscopy with endobronchial ultrasound (EBUS) was used to identify lymph nodes at multiple stations and perform lavage and biopsy.

\section{Diagnosis}

EBUS-guided biopsy of the lymph nodes revealed scattered lymphocytes, bronchial cells, negative for acid-fast bacilli (AFB), methenamine, or periodic-acid Schiff (PAS) reactivity for microorganisms and cellular changes suggestive of granulomas (Figs. 2-4). Bronchoalveolar lavage (BAL) revealed a few bronchial cells and numerous pulmonary macrophages. Left lower lobe transbronchial biopsy revealed non-caseating

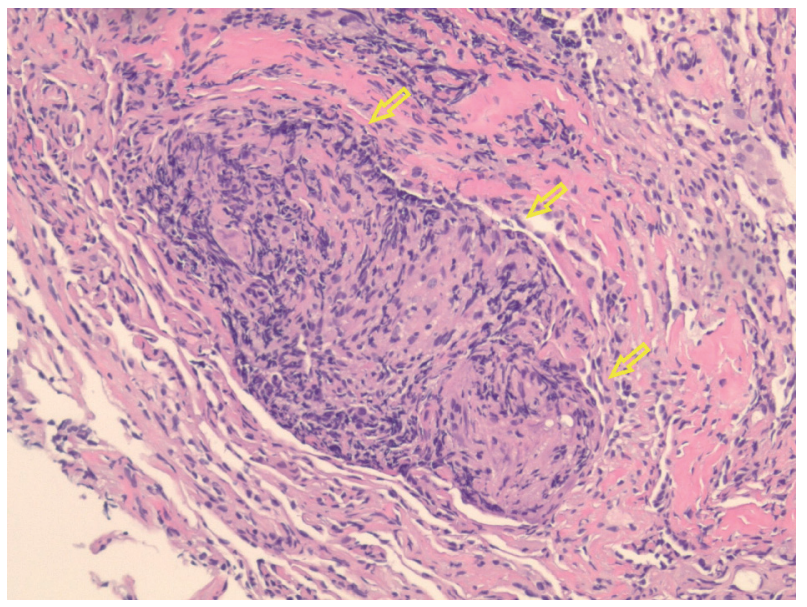

Figure 2. Hematoxylin and eosin-stained histological examination at $\times$ 20 magnification revealing non-caseating granulomas (arrows) in the left lower lung.

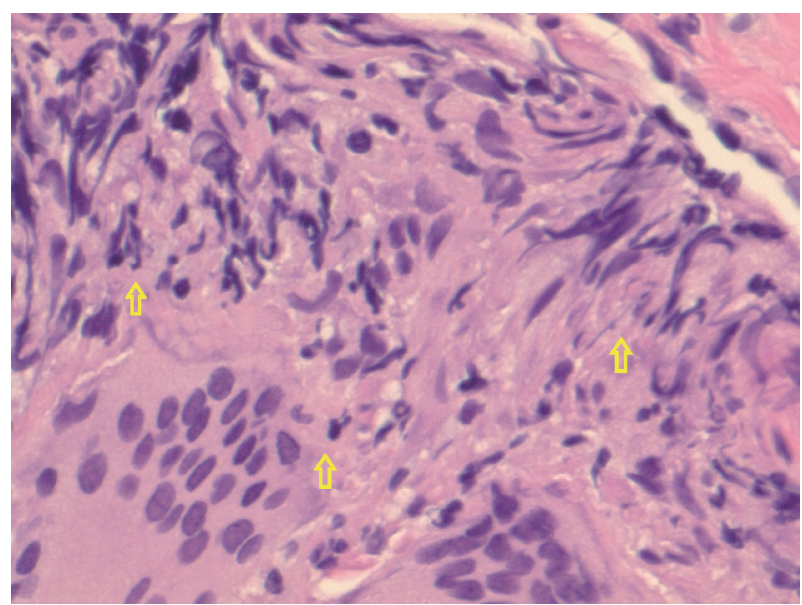

Figure 3. Hematoxylin and eosin-stained histological examination at $\times 40$ magnification revealing epithelioid histiocytes, lymphocytes, and multinucleated giant cells in the left lower lung.

granulomas with multinucleated giant cells with a reactive epithelium and no malignancy evidence. Subsequent liver biopsy pathology reports confirmed non-caseating granulomas and inflammation as per the pathologist report, as images could not be obtained. He was started on prednisone $20 \mathrm{mg}$ daily and ursodeoxycholic acid $300 \mathrm{mg}$ twice daily and required to follow-up monthly, and at his 3-month follow-up, he continued to endorse cough but denied worsening fatigue. A significant improvement in his biochemical profile was noted, and it was recommended he continue prednisone and follow-up with his pulmonologist and hepatologist in 3 months (Table 1).

\section{Discussion}

Sarcoidosis affects African Americans more commonly, and geographic epidemiological factors play a significant role, although specific factors have not been identified. Extrapulmo-

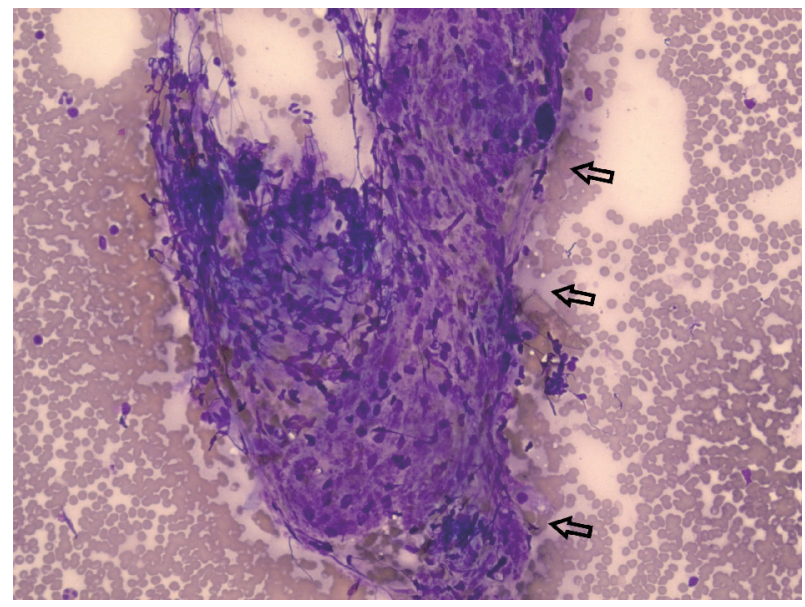

Figure 4. Diff-Quik stained histological examination at $\times 20$ magnification revealing epithelioid histiocytes and lymphocytes (arrows) from the left hilar lymph nodes. 


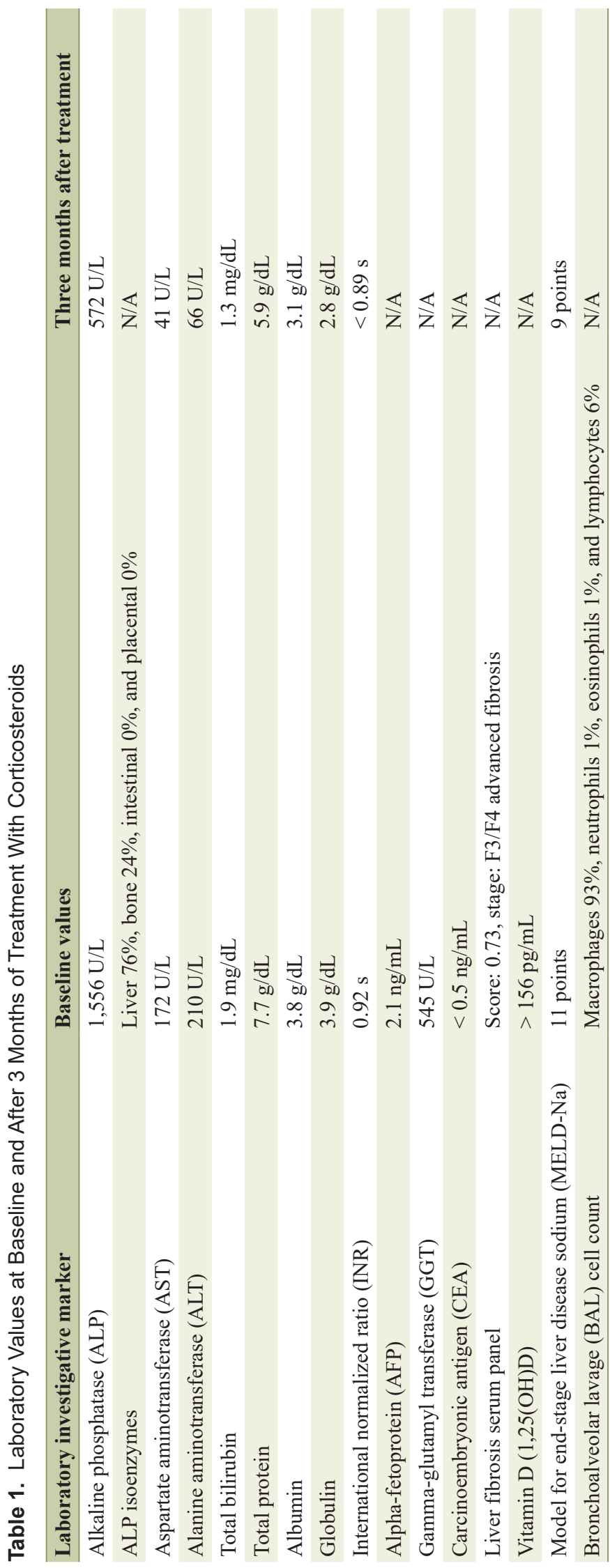

nary manifestations are rare, while consensus guidelines from the ATS recommend obtaining a baseline alkaline phosphatase level for screening purposes in newly diagnosed cases [3]. Ascites is less common in sarcoid-related portal hypertension than other causes and is transudative in nature. A significant distinction of portal hypertension from hepatic sarcoidosis is that cirrhosis is not always present; in fact, studies have seen it as low as $27 \%$, suggesting that the primary damage mechanism is progressive inflammation fibrosis. Granulomas can form anywhere within the sinusoids, walls of the veins, and parenchyma. Hepatosplenomegaly is seen in 50\% of presentations [5]. Clinical indicators are rare, and the majority remain asymptomatic. Pruritis and jaundice are seen in less than $7 \%$ of patients. This finding was consistent in our patient, with significant hyperbilirubinemia and without features of jaundice, possibly indicating that hepatic sarcoidosis involves a delayed presentation of clinical jaundice. Very few cases develop cirrhosis, and only less than $10 \%$ undergo orthotopic liver transplantation (OLT). Hepatic sarcoidosis makes up $0.10 \%$ of all OLT performed, and long-term survival rates are much worse in hepatic sarcoidosis than other cholestatic liver disease precipitants. In studies that demonstrated OLT's similar outcomes to control groups with no recurrence, the net protective effect may have been attenuated as patients were on post-transplant immunosuppression $[6,7]$.

Hepatocellular carcinoma (HCC) remains an ongoing risk in these patients, regardless of cirrhosis development, albeit the relationship is poorly understood. Laboratory abnormalities in aspartate aminotransferase (AST), alanine aminotransferase (ALT), gamma-glutamyl transferase (GGT) are all increased [8]. On ultrasound, hypoechoic nodules are characteristic, although hyperechoic findings may represent granulomas on the background of fibrosis. CT can discern contour irregularities, nodules, a confluence of granulomas, and parenchymal changes. T2weighted magnetic resonance imagining (MRI) can help parse out metastasis from granulomas, with lower signal intensities in granulomas. Hepatic sarcoidosis can affect any component of the liver, and liver injury patterns are broad. Apart from their destructive inflammatory effects, granulomas have compressive effects on the vascular system that can increase upstream pressures. A frequent confounder may be primary sclerosing cholangitis (PBC), and anti-mitochondrial antibodies (AMA) can help in this setting $[2,6,9]$. The mainstay of therapy, corticosteroids, has yielded mixed results, although improvements in liver function, portal hypertension gradients, nodule size, and ascites have been described. In a cohort of hepatic sarcoidosis patients $(\mathrm{n}=$ 49) treated with steroids for 14 days, significant improvements in biochemical and histological parameters were noted. Ursodeoxycholic acid (UDCA) has been widely used adjunctively, although no randomized trials of UDCA use in hepatic sarcoidosis patients exist. In steroid-refractory cases, methotrexate, azathioprine, and infliximab-based regimens have been adopted from pulmonary sarcoid research, but its risk of amplifying hepatotoxicity and bone marrow suppression limit the use of these agents. Progressive states eventually reach a state of end-stage liver disease and require transplantation [5, 10-12].

This case is limited by the lack of a histological image from the liver. Future studies can look into the role of alkaline phosphatase and its role as a surrogate marker for pulmonary sarcoidosis. 


\section{Learning points}

Hepatic sarcoidosis is exceedingly rare, but it can result in significant morbidity and mortality when it does present. A combination of imaging and histology clinches the diagnosis, and newly diagnosed patients with sarcoidosis should receive an alkaline phosphate screening. Progressive disease can lead to cirrhosis or hepatocellular carcinoma. Established hepatic sarcoidosis should undergo periodic surveillance and consider transaminitis in the right setting as evidence of disease exacerbation.

\section{Acknowledgments}

We would like to thank our library manager, Jennifer Godlesky, for help with a literature search on this topic and Dr. Amro Almradi (consultant pathologist) for interpreting histologic specimens.

\section{Financial Disclosure}

None to declare.

\section{Conflict of Interest}

None to declare.

\section{Informed Consent}

Obtained.

\section{Author Contributions}

Smit S. Deliwala: conception, draft, and review. Murtaza Hussain: acquisition and draft. Anoosha Ponnapalli: acquisition and draft. Rupesh Khanal: draft and review. Hemant Goyal: draft and review. Adil Abdalla: draft and review. Mamoon M. Elbedawi: draft and review.

\section{Data Availability}

The authors declare that data supporting the findings of this study are available within the article.

\section{References}

1. Arkema EV, Cozier YC. Epidemiology of sarcoidosis: current findings and future directions. Ther Adv Chronic Dis. 2018;9(11):227-240.

2. Tadros M, Forouhar F, Wu GY. Hepatic Sarcoidosis. J Clin Transl Hepatol. 2013;1(2):87-93.

3. Ungprasert P, Crowson CS, Simonetto DA, Matteson EL. Clinical characteristics and outcome of hepatic sarcoidosis: a population-based study 1976-2013. Am J Gastroenterol. 2017;112(10):1556-1563.

4. Crouser ED, Maier LA, Wilson KC, Bonham CA, Morgenthau AS, Patterson KC, Abston E, et al. Diagnosis and detection of sarcoidosis. An official American thoracic society clinical practice guideline. Am J Respir Crit Care Med. 2020;201(8):e26-e51.

5. Sedki M, Fonseca N, Santiago P, Diaz L, Garcia-Buitrago M, Mirsaeidi M, Levy C. Hepatic sarcoidosis: natural history and management implications. Front Med (Lausanne). 2019;6:232.

6. Bilal M, Satapathy SK, Ismail MK, Vanatta JM. Longterm outcomes of liver transplantation for hepatic sarcoidosis: a single center experience. J Clin Exp Hepatol. 2016;6(2):94-99.

7. Vanatta JM, Modanlou KA, Dean AG, Nezakatgoo N, Campos L, Nair S, Eason JD. Outcomes of orthotopic liver transplantation for hepatic sarcoidosis: an analysis of the United Network for Organ Sharing/Organ Procurement and Transplantation Network data files for a comparative study with cholestatic liver diseases. Liver Transpl. 2011;17(9):1027-1034.

8. Arai T, Akita S, Sakon M, Fujii T, Tanaka H, Ishii K, Miwa S. Hepatocellular carcinoma associated with sarcoidosis. Int J Surg Case Rep. 2014;5(8):562-565.

9. Deutsch-Link S, Fortuna D, Weinberg EM. A comprehensive review of hepatic sarcoid. Semin Liver Dis. 2018;38(3):284-297.

10. Ayyala US, Padilla ML. Diagnosis and treatment of hepatic sarcoidosis. Curr Treat Options Gastroenterol. 2006;9(6):475-483.

11. Cremers JP, Drent M, Baughman RP, Wijnen PA, Koek GH. Therapeutic approach of hepatic sarcoidosis. Curr Opin Pulm Med. 2012;18(5):472-482.

12. El Jammal T, Jamilloux Y, Gerfaud-Valentin M, Valeyre D, Seve P. Refractory sarcoidosis: a review. Ther Clin Risk Manag. 2020;16:323-345. 